\title{
Effectiveness of interventions designed to promote patient involvement to enhance safety: a systematic review
}

\author{
Jill Hall, ${ }^{1}$ Maggie Peat, ${ }^{1}$ Yvonne Birks, ${ }^{1}$ Su Golder, ${ }^{1}$ on behalf of the PIPS Group, \\ Vikki Entwistle, ${ }^{2}$ Simon Gilbody, ${ }^{1}$ Peter Mansell, ${ }^{3}$ Dorothy McCaughan, ${ }^{1}$ \\ Trevor Sheldon, ${ }^{1}$ lan Watt, ${ }^{1}$ Brian Williams, ${ }^{2}$ John Wright ${ }^{4}$
}

\begin{abstract}
${ }^{1}$ Hull York Medical School, University of York, York, UK University of Dundee, Dundee, UK

${ }^{3}$ National Patient Safety Agency, London, UK ${ }^{4}$ Bradford Teaching Hospitals NHS Foundation Trust, Bradford, UK
\end{abstract}

\section{Correspondence to}

Mrs Jill Hall, University of York, Department of Health Sciences, Area 2 Seebohm Rowntree Building, Heslington, York Y010 5DD, UK; jh545@york.ac.uk

Patient Involvement in Patient Safety (PIPS) Group: VE, SG, PM, DM, TS, IW, BW, JW.

Accepted 27 June 2009 Published Online First 27 April 2010

\begin{abstract}
Background There is growing international interest in involving patients in interventions to promote and support them in securing their own safety. This paper reports a systematic review of evaluations of the effectiveness of interventions that have been used with the explicit intention of promoting patient involvement in patient safety in healthcare.
\end{abstract}

Methods The authors searched Cochrane Database of Systematic Reviews, Database of Abstracts of Reviews of Effects, CENTRAL, CINAHL, EMBASE, HMIC,

MEDLINE, MEDLINE in-process, PsycINFO and ASSIA to August 2008. We also searched databases of reports, conference proceedings, grey literature, ongoing research and relevant patient safety organisations, and hand-searched two journals. Meta-analysis of the data was not appropriate; therefore, studies were categorised according to how the interventions encouraged patients' actions to improve safety-informing the management plan, monitoring and ensuring safe delivery of treatment (by health professional and by self), making systems safer-and were critiqued in a narrative manner.

Findings The authors identified 14 individual experimental and quasiexperimental studies plus one systematic review. The majority of studies fell into the monitoring and ensuring safe delivery of treatment by self category and were all related to enhancing medication safety. Authors reported improved patient safety incident outcomes for the intervention groups compared with controls where the interventions aimed to encourage patient involvement in: (1) monitoring and ensuring safe delivery of treatment by self (self-management of anticoagulation, 'easy' read information leaflet, nurse-led education to promote selfmedication in hospital, patient package insert using lay terminology); (2) informing the management plan/ monitoring and ensuring safe delivery of treatment by self (individualised teaching plan by nurse, pharmacist counselling). It was not possible to draw any clear conclusions as to the effectiveness of the interventions (with the exception of one specific aspect of self-medication, that is, self-management of anticoagulation) due to concerns about the methodological quality of the studies.

Conclusions There is limited evidence for the effectiveness of interventions designed to promote patient involvement on patient safety incidents and in general is poor quality. Existing evidence is confined to the promotion of safe selfmanagement of medication, most notably relating to the self-management of oral anticoagulants.

\section{BACKGROUND}

International estimates suggest that between 3\% and $17 \%$ of hospital admissions result in an adverse event and that between $28 \%$ and $75 \%$ of them are preventable. ${ }^{1}$ Strategies to reduce adverse events have focused mainly on the change of systems of care and professional behaviour. However, more recently, there has been growing international interest in the development and use of interventions to promote and support patients' (and their family members' or advocate) roles in securing their own safety in healthcare contexts.

The provision of safety-related advice, in the form of a 'tip sheet,' is the most common intervention currently used by healthcare providers that aim to encourage patients to contribute to their own safety. For instance, '20 tips to help prevent medical errors' (USA) and 'Ask About Your Medicines' (Croatia). ${ }^{2} 3$ Another method by which patients may contribute to improved safety (both their own and others), is through participation in reporting systems-for example, Meldpunt Medicijnen (DGV) in The Netherlands. ${ }^{4}$ Previous research suggests that while these interventions hold potential, there is scant information on their effectiveness. ${ }^{56}$

The concept of patient involvement in healthcare is not unique to the area of patient safety. Indeed, there is a broad interest and literature base for patient involvement strategies in healthcare more generally; however, this paper reports the first systematic review of the research evidence on the effectiveness of interventions designed to promote patient involvement specifically to enhance safety, in a healthcare context.

\section{OBJECTIVES}

To identify, appraise and summarise evaluations of strategies or interventions which have been used with the explicit intention of promoting patients' (and/or their family members' or advocates') involvement in their care with a view to enhancing their own, or others' safety in a healthcare context.

\section{METHODS}

\section{Eligibility and search strategy}

We included all published and unpublished systematic reviews, experimental studies and quasiexperimental studies that evaluated any intervention which promoted or supported patients' involvement (and/or their family/representatives), in activities 
relating to their healthcare with the explicit intention of enhancing patient safety. Experimental studies were defined as 'participants allocated to intervention or control groups by means of randomisation,' and quasiexperimental studies were defined as 'allocation to groups is under the control of the investigator but falls short of genuine randomisation.' We included any health service users or potential health service users in any healthcare context. Systematic reviews were included provided they were of a high quality and recently published. We excluded studies that promoted or supported patients in activities relating to their healthcare, but did not explicitly aim to enhance safety (for example, in-patient self-medication which aimed to improve pain control or, coaching and question prompts which aimed to improve clinical decision-making). Outcomes of interest were patient safety incidents such as adverse incidents, adverse events, near misses, medication error rates and infection rates.

This review was conducted in conjunction with a broadranging review of strategies to promote patient involvement to enhance safety. Therefore, the search strategies were broad in nature in order to capture relevant records for each of the literature reviews. A range of free text terms and subject headings were used for both the patient involvement concept of the question and the patient safety element. Key terms for patient involvement included terms to describe 'patient' (eg, consumer, citizen, public, carer, care giver, user), terms to describe 'involvement' (eg, view, attitude, role, contribution, partner, engagement, opinion) and subject headings (consumer participation, patient education). Key terms for patient safety included risk, safe, mistake, error, near miss, adverse reporting and subject headings such as safety management, risk management, medical errors and medication errors. There were no language restrictions. We searched Cochrane Database of Systematic Reviews (CDSR) (Issue 3, 2008), Database of Abstracts of Reviews of Effects (DARE) (July 2008), CENTRAL (Issue 3, 2008), CINAHL (1982 to July 2008), EMBASE (1980 to Week 29 2008), HMIC Health Management Information Consortium (July 2008), MEDLINE (1966 to July 2008), MEDLINE In-process \& other non-indexed citations (July 2008), PsycINFO (1967 to July 2008), Applied Social Sciences Index and Abstracts (ASSIA) (1987 to July 2008) and NHS Economic Evaluations Database (NHS EED) (July 2008). We also searched databases of reports, conference proceedings, grey literature and ongoing research. We sought additional studies from: the websites of patient safety organisations, hand-searched two specialist patient safety journals and consulted topic specialists within the research team.

\section{Selection of studies and data extraction}

Two authors screened citations of titles and abstracts for potentially relevant papers. The inclusion criteria were then applied independently (based on the full paper) by two authors and data extracted according to predefined criteria. Disagreements were resolved by consultation with a third author. We assessed the methodological quality of all included studies. Systematic reviews were assessed according to the Quality of Reporting of Meta-analyses (QUOROM) statement. ${ }^{7}$ Experimental and quasiexperimental studies were assessed according to criteria recommended by the Centre for Reviews and Dissemination (CRD). ${ }^{8}$

\section{Data analysis}

After looking at the included studies in terms of participants, interventions and outcomes, it was not considered appropriate to undertake any formal pooling of data.

\section{RESULTS}

\section{Results of the search}

Over 22000 references were retrieved by the searches (figure 1). Sixty-eight references were identified as potentially relevant. A total of 15 studies met the eligibility criteria. Four additional papers were identified as copublications that reported aspects of the same study. To date, one paper has not been received and awaits classification. The search did not identify any ongoing studies.

There were 14 individual studies with 8460 participants (although there were 17270 participants prior to postrandomisation exclusions) and one systematic review (included 16 RCTs) (table 1). The systematic review also included a review of non-randomised controlled studies and is not reported here because a number of the study designs did not fit the inclusion criteria for this review.

\section{Design}

Among the 14 individual studies included in the present review, 11 were an experimental design, and the remainder were quasiexperimental. 91122 The majority of the individual studies were conducted in the USA $(n=9)$, plus one in Canada, ${ }^{18}$ one in Australia, ${ }^{9}$ one in Nepal, ${ }^{15}$ one in Belgium ${ }^{21}$ and one in the UK. ${ }^{14}$ The length of follow-up in these 14 individual trials ranged from immediately following the intervention ${ }^{15} 16$ up to 12 months. ${ }^{9}$ In the systematic review, the duration of the included studies varied from 2 months to 24 months. ${ }^{10}$

\section{Setting}

Among the individual studies included in the present review, eight took place in a hospital setting (five ward-based and three in outpatients), three in general practice clinics 92122 and three in other community settings. ${ }^{16} 1719$

\section{Participants}

The majority of individual studies in the present review included adult participants. ${ }^{11} 14 \quad 15$ 19-23 Five studies included elderly participants. ${ }^{9} 12131718$ One study recruited parents of paediatric patients. ${ }^{16}$ The mean age of the participants varied from approximately 38 years to 80 years, and the proportion of male participants in the studies ranged from $12 \%$ to $55 \%$. In the systematic review, the mean age of participants ranged from 42 to 75 years. $^{10}$

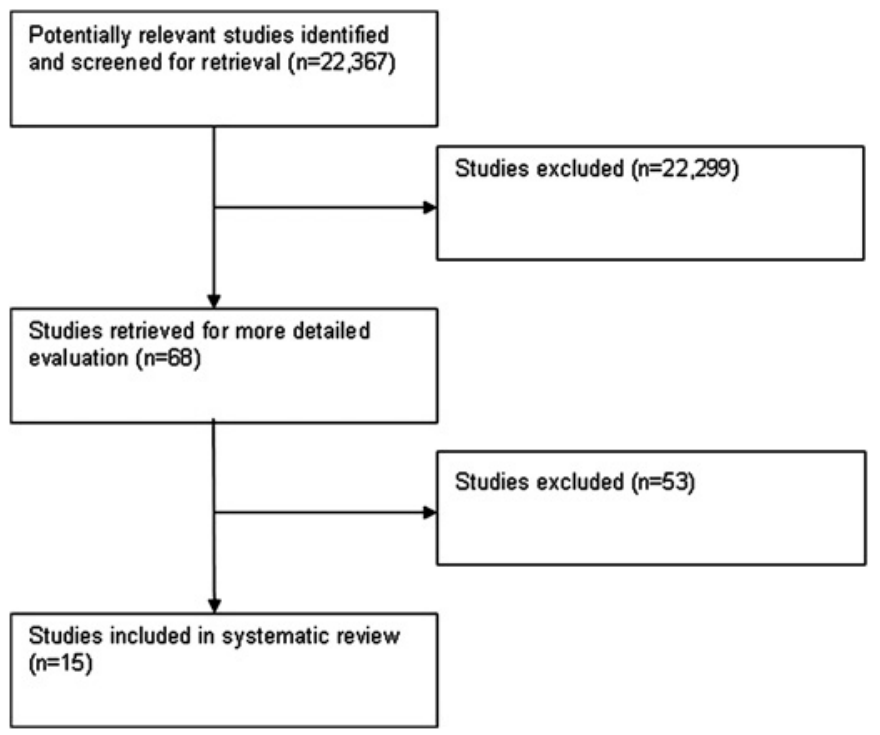

Figure 1 Flow chart of identification of relevant studies. 


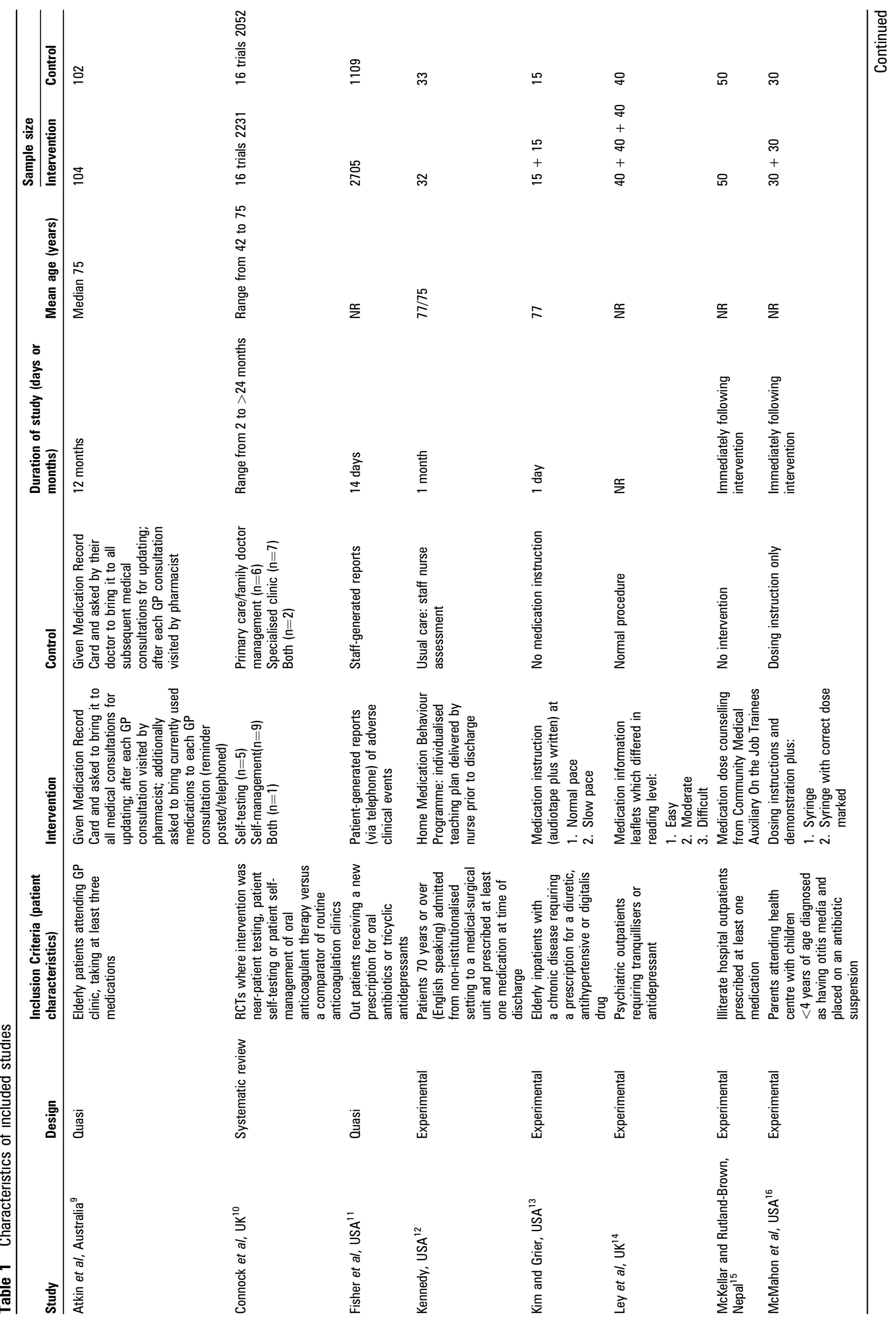




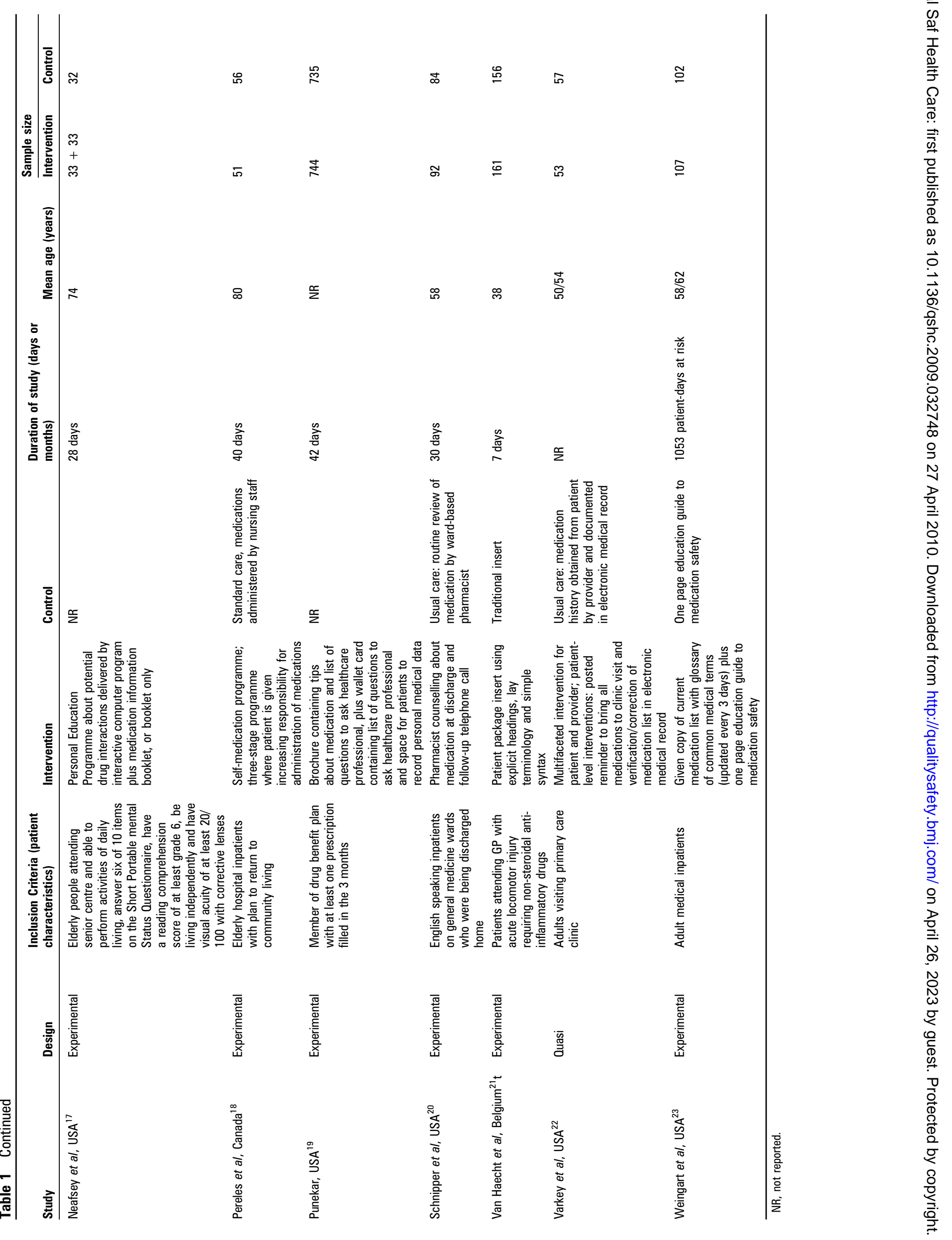




\section{Interventions}

To gain a better understanding of the interventions included in this review, three broad routes by which patients' actions might contribute to safety were identified. The categorisations were originally developed (and described in further detail) for a broadranging review of patient involvement in safety, ${ }^{24}$ which was undertaken in conjunction with this systematic review. The categorisations were:

1. informing the management plan: helping to ensure the appropriate treatment plan is formulated (eg, patients make sure that healthcare professionals have information about any of their allergies or adverse reactions to medication);

2. monitoring and ensuring safe delivery of treatment: helping to ensure the management plan is correctly implemented by:

(a) helping to ensure safe delivery of planned treatment by health professionals (eg, checking that the correct dose of chemotherapy medication is administered at the right time); and (b) helping to ensure safe delivery of treatment by self (eg, patient self management of anticoagulation treatment);

3. making systems safer: helping ensure that current and future healthcare systems are safe (eg, patients acting as patient representatives on a hospital safety committee).

These are not completely mutually exclusive. For example, patient use of a treatment diary could be categorised as informing the management plan if the intention was that patients complete and update the diary with information about themselves. In addition, the diary could also be categorised as supporting monitoring and ensuring safe delivery of treatment if it contained general information for warning signs and symptoms to look out for and list routine tests that should be carried out.

Indirectly, all interventions could contribute to making systems safer, but for the purpose of this review, only those which explicitly set out to involve patients in ways that would have impacts on patient safety beyond the scope of their own care have been categorised as such.

The interventions employed in the 14 studies and one systematic review were all related to the use of medications and were classified as follows:

1. Eight individual studies and the systematic review reported on interventions that encouraged patients in monitoring and ensuring safe delivery of treatment. ${ }^{10} 13-1618192123$ In one study, the intervention was concerned with helping to ensure safe delivery of planned treatment by health professionals. ${ }^{23}$ The remainder were helping to ensure safe delivery of treatment by self.

2. In two studies, the interventions were classified as 'informing the management plan.'9 22

3. In three studies, the interventions were classified as 'informing the management plan combined with monitoring and ensuring safe delivery of treatment (by self)., ${ }^{12} 1720$

4. In one study, the intervention was classified as informing the management plan combined with making systems safer.' ${ }^{, 11}$

\section{Outcomes}

Seven of the 14 individual studies plus the systematic review reported at least one outcome related to patient safety incidents. ${ }^{10-12} 14 \quad 18202123$ These included death, medication errors, adverse drug events and reactions, close-call drug errors and patient reporting of adverse clinical events.

\section{Methodological quality of included studies}

Overall, the methodological quality of the majority of the included individual studies was poor. For example, none of the 11 experimental studies provided details regarding concealment of allocation, and only four (of the experimental and quasiexperimental) studies provided details of blinded outcome assessment. The quality of the systematic review and meta-analysis of self-management of anticoagulation was good, addressing all the items on the QUOROM checklist.

\section{Effects of interventions}

In the two studies where the intervention aimed to involve patients by their 'informing the management plan,' no patient safety incident outcomes were reported. In the study categorised as 'monitoring and ensuring safe delivery of treatment by health professional' and also in the study categorised as 'informing the management plan/making systems safer,' there were no differences in outcomes between the intervention and control groups. In the category informing the management plan/monitoring and ensuring safe delivery of treatment by self, two studies reported favourable outcomes for the intervention group compared with the control (one study in this category did not report any patient safety incident outcomes). In the eight studies where the interventions aimed to involve patients in 'monitoring and ensuring safe delivery of treatment by self,' four of them reported patient safety incident outcomes and in the main describe improved outcomes for the intervention groups compared with controls (table 2).

\section{DISCUSSION}

This review identified evidence of safety benefit for patient involvement in one specific aspect of self-medication but little evidence of effectiveness in other aspects of healthcare.

The majority of studies fell into the monitoring and ensuring safe delivery of treatment by self category, and the interventions were all related to enhancing medication safety. Only half of the included studies evaluated at least one outcome related to patient safety incidents. Of those that did evaluate patient safety incidents, authors reported improved outcomes for the intervention groups compared with controls where the interventions aimed to encourage patient involvement in:

1. monitoring and ensuring safe delivery of treatment by self (self-management of anticoagulation, 'easy' read information leaflet, self-medication in hospital, patient package insert using lay terminology);

2. informing the management plan/monitoring and ensuring safe delivery of treatment (individualised teaching plan by nurse, pharmacist counselling).

However, it was not possible to draw any clear conclusions as to the effectiveness of the interventions (with the exception of one specific aspect of self-medication, ie, self-management of anticoagulation) due to concerns about the methodological quality of the studies.

The evidence identified in our review does not sufficiently address all potential areas of patient involvement in patient safety. For instance, the included studies and interventions were all pertaining to patient involvement to ensure medication safety, especially by promoting safe self-medication. We found no robust evaluations of efforts to involve patients in other areas of healthcare and only one evaluation encouraging patients to monitor professionally delivered healthcare. In particular, there are no evaluations of the many 'advisories' offering 'hints and tips' to patients about how to ensure they stay safe when using healthcare services, and no high-profile initiatives such as the patient empowerment element of the UK-based 'Clean your hands' campaign (although ongoing work by the NPSA aims to provide 'evidence of robust evaluation' in the future). ${ }^{25}$ 
Table 2 Effect of interventions on patient safety incident outcomes

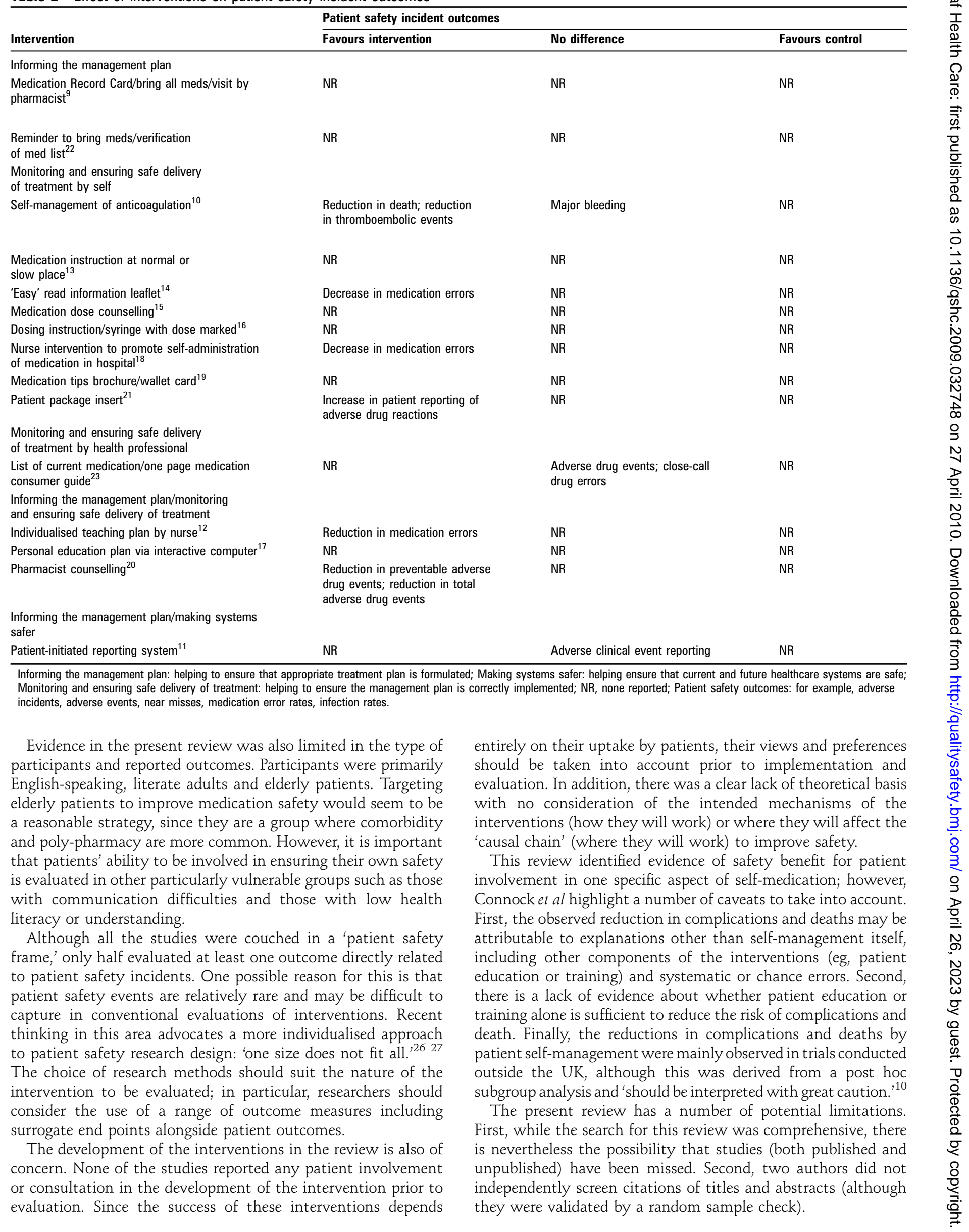


Third, to be included in this review, studies had to be couched in a 'patient safety frame,' explicitly stating that their aim was to improve safety. Many similar interventions that involve patients may, indirectly, also impact on safety. However, any studies of these that were not reported as evaluations of safetypromoting activities may not have been identified by our search strategy and would not have fulfilled our inclusion criteria. For instance, we are aware of a body of literature pertaining to interventions that involve patients with the aim of enhancing medication adherence. A recent Cochrane review of this literature selected RCTs that reported an intervention to improve medication adherence and treatment outcome. ${ }^{28}$ The review included a total of 93 interventions, of which many were very similar to those identified in our review. For example, more instruction or counselling for patients, involving patients in selfmonitoring of blood pressure, dose-dispensing units and posted communications to patients. The findings from the Cochrane review suggest that 'the literature concerning interventions to improve adherence with medications remains surprisingly weak' with little evidence that medication adherence can be improved consistently and lead to improvements in treatment outcomes.

Finally, this review focussed on effectiveness of the interventions. We did not attempt to summarise the 'process' of patient involvement in patient safety; however, we recognise that this is an important consideration in evaluations of patient-involvement strategies. A scoping review of interventions intended to involve patients in patient safety, which was conducted in conjunction with this systematic review, found that patients have, largely, not been involved in the development of interventions, and little is known about their willingness and ability to adopt recommended patient-safety-promoting behaviours. ${ }^{24}$ Building on the findings of that review, we developed an approach to the appraisal of interventions which encourages attention to the mechanisms by which patients might contribute to their safety, the conditions under which their contributions are likely to be successful and the extent to which these interventions are likely to ensure those conditions are filled in particular contexts. Incorporating this type of appraisal and qualitative observations (of patients' views and attitudes) within evaluations of interventions can assist in illuminating the processes whereby patients may be involved in enhancing safety.

\section{CONCLUSIONS}

There is a major international movement to increase patient involvement with a view to enhancing patient safety. However, there is scarce evidence of benefit. This review identified evidence of safety benefit for patient involvement in one specific aspect of self-medication, but little evidence of effectiveness in other aspects of healthcare. Future research should focus on areas other than medication safety. Those undertaking future research should carefully consider what is the most appropriate research design for the intervention or strategy to be evaluated. In particular, what outcomes should be measured and the inclusion of qualitative methods to complement and illuminate the assessment of patient-involvement strategies.

Funding This research was funded by the Department of Health, Patient Safety Research Portfolio, UK.

\section{Competing interests None.}

Provenance and peer review Not commissioned; externally peer reviewed.

\section{REFERENCES}

1. Sari AB, Sheldon TA, Cracknell A, et al. Extent, nature and consequences of adverse events: results of a retrospective casenote review in a large NHS hospital. Qual Saf Health Care 2007;16:434-9.
2. AHR0. 20 tips to help prevent medical errors. Rockville, MD: Agency for Healthcare Research and Quality, 2000. http://www.ahrq.gov/consumer/20tips.htm (accessed Nov 2008).

3. Jaksevac-Miksa M, Curic J. 'Ask About Your Medicines'—Patient Education Project. Pharmacy and Pharmaceutical Sciences World Congress, 2002. Abstract 218.

4. Meldpunt Medicijnen, Netherlands. http://www.meldpuntmedicijnen.nl/ (accessed June 2009).

5. Pizzi LT, Goldfarb NI, Nash DB. Other practices related to patient participation. In Shojania KG, Duncan BW, McDonald KM, et al, eds. Making health care safer: a critical analysis of patient safety practices. Rockville, MD: Agency for Healthcare Research and Quality. http://www.ahrq.gov/clinic/ptsafety/ (accessed Nov 2008).

6. Entwistle VA, Mello MM, Brennan TA. Advising patients about patient safety: current initiatives risk shifting responsibility. Jt Comm J Qual Patient Saf 2005;31:483-94.

7. Moher D, Cook DJ, Eastwood S, et al. Improving the quality of reports of metaanalyses of randomised controlled trials: the QUOROM statement. Lancet 1999:354:1896-900.

8. CRD. Undertaking Systematic Reviews of Research on Effectiveness. CRD's guidance for those carrying out or commissioning reviews. CRD Report Number 4. 2nd edn, 2001. http://www.york.ac.uk/inst/crd/report4.htm (accessed Nov 2008).

9. Atkin P, Stringer RS, Duffy JB, et al. The influence of information provided by patients on the accuracy of medication records. Med J Aust 1998;169:85-8.

10. Connock M, Stevens C, Fry-Smith A, et al. Clinical effectiveness and costeffectiveness of different models of managing long-term oral anticoagulation therapy: a systematic review and economic modelling. Health Technol Assess 2007:11:iii-iv.

11. Fisher S, Bryant SG, Solovitz BL, et al. Patient-initiated post marketing surveillance: a validation study. J Clin Pharmacol 1987:27:843-54.

Co-publications for Fisher 1987 Fisher S, Bryant SG, Kluge RM. Detecting adverse drug reactions in post marketing surveillance: interview validity. Drug Inf J 1987;21:173-83. Fisher S, Bryant SG. Post marketing surveillance: accuracy of patient drug attribution judgments. Clin Pharmacol Ther 1990;48:102-7.

12. Kennedy LM. The effectiveness of a self-care medication education protocol on the home medication behaviors of recently hospitalized elderly. The University of Texas at Austin. (0227), 1990.

13. Kim KK, Grier MR. Pacing effects of medication instruction for the elderly. J Gerontol Nurs 1981;7:464-8.

14. Ley $\mathbf{P}$, Jain VK, Skilbeck CE. A method for decreasing patients' medication errors Psychol Med 1976:6:599-601.

15. McKellar AT, Rutland-Brown W. Using community medical auxiliary trainees to improve dose understanding among illiterate hospital outpatients in rural Nepal. Trop Doct 2005;35:17-18.

16. McMahon SR, Rimsza ME, Bay RC. Parents can dose liquid medication accurately. Pediatrics 1997; 100:330-3.

Co-publication for McMahon 1997. Anonymous. Educating parents, providing measuring devices could eliminate pediatric dosage errors, researchers show. Am $J$ Health Syst Pharm 1997:54:2289-90.

17. Neafsey PJ, Strickler Z, Shellman J, et al. An interactive technology approach to educate older adults about drug interactions arising from over-the-counter self-medication practices. Public Health Nurs 2002:19:255-62.

18. Pereles L, Romonko L, Murzyn T, et al. Evaluation of a self-medication program J Am Geriatr Soc 1996;44:161-5.

19. Punekar YS. Development and validation of a patient medication risk reduction behavior scale and application in a managed care population. Purdue University, 2003:190.

20. Schnipper JL, Kirwin JL, Cotugno MC, et al. Role of pharmacist counselling in preventing adverse drug events after hospitalization. Arch Intern Med 2006;166:565-71.

21. Van Haecht CHM, Vander Stichele R, De Backer G, et al. Impact of patient package inserts on patients' satisfaction, adverse drug reactions and risk perception: The case of NSAIDs for posttraumatic pain relief. Patient Educ Couns 1991;17:205-15.

22. Varkey $\mathbf{P}$, Cunningham J, Bisping S. Improving medication reconciliation in the outpatient setting. Jt Comm J Qual Patient Saf 2007;33:286-92.

23. Weingart SN, Toth $M$, Eneman J, et al. Lessons from a patient partnership intervention to prevent adverse drug events. Int J Qual Health Care 2004;16:499-507. Co-publication for Weingart 2004. Partnering with hospitalized patients to monitor medication use is a feasible strategy for reducing drug errors. Research Activities 2005;298:18-19.

24. Peat M, Entwistle V, Hall J, et al. on behalf of the PIPS group. Scoping review and approach to appraisal of interventions intended to involve patients in patient safety. Journal of Health Services Research \& Policy 2010;15:17-25.

25. National Patient Safety Agency. Clean your hands campaign. Patient empowerment pilot. http://www.npsa.nhs.uk/cleanyourhands/in-hospitals/pep/ (accessed December 2008).

26. Brown C, Hofer T, Johal A, et al. An epistemology of patient safety research: a framework for study design and interpretation. Part 4. One size does not fit all. Qual Saf Health Care 2008;17:178-81.

27. Brown C, Hofer T, Johal A, et al. An epistemology of patient safety research: a framework for study design and interpretation. Part 3. End points and measurement. Qual Saf Health Care 2008;17:170-7.

28. Haynes RB, Ackloo E, Sahota N, McDonald HP, Yao X. Interventions for enhancing medication adherence. Cochrane Database of Syst Rev 2008, Issue 2. Art. No. CD000011 